\title{
A Challenging Case of Spontaneous Coronary Artery Dissection
}

\author{
Abhisekh Mohanty \\ DM cardiology \\ Department of Cardiology, Continental Hospitals, Hyderabad, India \\ abhisekh.mhnt@gmail.com
}

\begin{abstract}
Spontaneous coronary artery dissection (SCAD) is a rarely reported cause of myocardial infarction $(M I)$, arrhythmias and sudden cardiac death (SCD). It predominantly affects young women. The pathological mechanism has not been fully clarified yet. Most of such cases have been attributed to fibromuscular dysplasia (FMD). However, several other diseases and conditions have been associated with SCAD, such as atherosclerosis, other connective tissue disorders and the peripartum state. In this paper we present a case of a 35 year old male presenting with ST segment elevation myocardial infarction (STEMI) due to coronary artery dissection and how we managed the case. We will also review the literature, discussing the possible mechanisms for SCAD, therapeutic options and prognosis.
\end{abstract}

Keywords: Spontaneous coronary artery dissection, myocardial ischemia.

\section{INTRODUCTION}

Spontaneous coronary artery dissection (SCAD) has been reported to be a rare cause of acute coronary syndrome (ACS) and sudden cardiac death (SCD). It is well known that SCAD is underdiagnosed and might be more prevalent than previously reported. It has a striking predilection for young females. The patho-physiology and the management of such cases is still a matter of debate. In this article we aim to discuss a similar case.

\section{CASE Report}

A 35 year old male, who is a smoker with no additional co-morbidities, presented to us with a history of angina on exertion since last 1 month. He was diagnosed as a case of Anterior wall ST segment elevation myocardial infarction ( STEMI) 1 month back. He was initially treated in a primary health care centre with anticoagulants, dual antiplatelet therapy and beta-blockers. When he came to us, he was hemodynamically stable. His echocardiogram was showing regional wall motion abnormality of the left ventricular apex and interventricular septum. We did a coronary angiogram which showed a long dissected segment starting from the proximal segment of left anterior descending( LAD) and extending till mid LAD after which it was totally occluded ( FIGURE 1 ). The rest of his coronary and peripheral vasculatures were completely normal. Since it was a case of STEMI, we decided to do percutaneous coronary intervention (PCI) . Initially we faced difficulty in crossing the lesion with a guidewire. We used a hydrophilic wire and a $1.25 \mathrm{~mm}$ diameter semicompliant balloon for additional support to cross the lesion. Then we used a long stent covering the dissected segment and also extending $5 \mathrm{~mm}$ proximally and distally. We achieved good angiographic result with TIMI 3 flow.

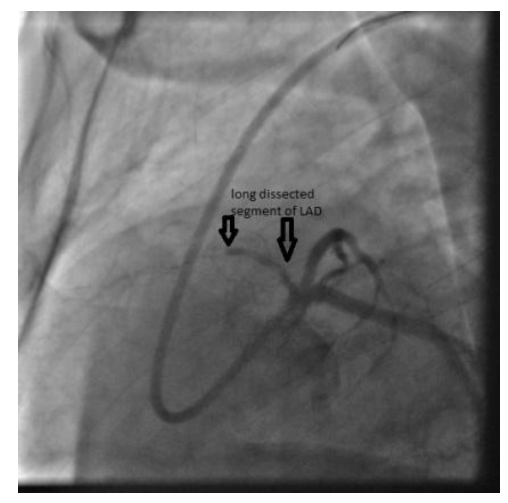

Figure1. Left Anterior Oblique (LAO) caudal view of left coronary artery showing dissected segment of proximal to mid LAD 
After 6 months, one follow up coronary angiogram was done which showed a patent stent and normally flowing epicardial coronary arteries ( FIGURE 2 ).

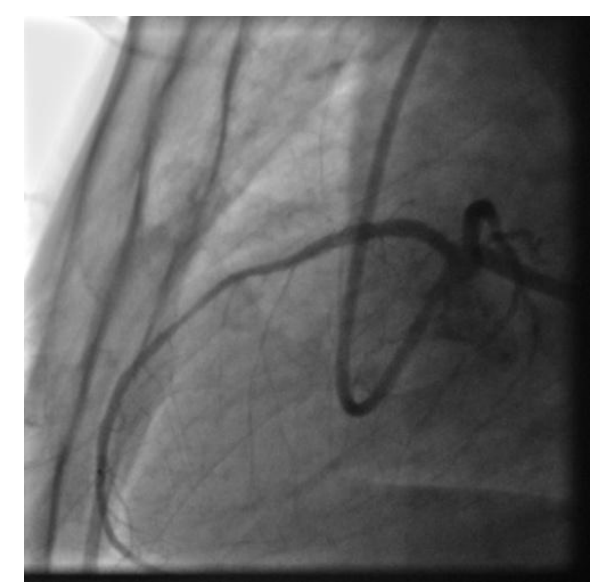

Figure2. LAO caudal view of left coronary artery done after 6 months of stenting of LAD showing patent artery

\section{DISCUSSION}

SCAD has been reported to be a rare cause of acute coronary syndrome (ACS) and sudden cardiac death (SCD). The first autopsy report involved a 42-year-old woman with SCD in 1931. It is difficult to distinguish between dissection and atherosclerotic obstruction. A high index of suspicion is required to diagnose it. Since it is under-reported, the exact incidence and outcomes, pathophysiology, and management are still a matter of debate . A collection of case reports from 1980-2000 showed mortality rates ranging from $0 \%$ to $7 \%{ }^{1}$. The mean age at presentation is 35 to 40 years and more than $70 \%$ of SCAD cases are women.

Although the pathological mechanism is not fully clarified, several diseases and conditions have been associated with $\mathrm{SCAD}^{2}$. It is thought to be the consequence of an intramural haematoma(IMH) of a coronary artery, resulting in a false lumen which compresses the true lumen, with subsequent myocardial ischaemia. The predisposing underlying mechanism may be atherosclerosis or certain non-atherosclerotic conditions like connective tissue disorders and peripartum state. In the majority of cases, an underlying condition which may lead to SCAD cannot be identified at all. Such cases are subsequently classified as 'idiopathic' ${ }^{2}$. Non-atherosclerotic SCAD predominantly affects women, and most of these patients have Fibromuscular Dysplasia (FMD) involving at least 1 non-coronary territory. The diagnosis of FMD can be confirmed only by biopsy studies, which was not done in our case. The etiology in our case was most likely non-atherosclerotic as he is a young patient with no additional co-morbidities and no other angiographic signs of atherosclerosis. Since he is a male patient with a history of smoking and rest of his vasculatures were completely normal, we think that he may be classified a case of 'idiopathic' SCAD.

Currently, clinical diagnosis of SCAD relies on the visualization of a radiolucent intimal "flap" on coronary angiography. Intravascular ultrasound (IVUS) and Optical coherence tomography (OCT) give a better picture of IMH and dissections. They also help during PCI to exactly localize the edges of dissection ${ }^{3}$.

The role of anticoagulation and thrombolytic therapy for SCAD is controversial with the risk of dissection extension balanced by the potential benefit of resolving overlying thrombus and improving true lumen patency. Thus, early coronary angiography is important if SCAD is suspected because medical management deviates from standard ACS therapy. However, thrombolysis should not be withheld for STEMI patients since SCAD is rare . Beta-blockers reduce arterial shear stress and are presumably beneficial in reducing coronary arterial wall stress, similar to the benefits in aortic dissection. The use of statins and angiotensin inhibitors for non-atherosclerotic SCAD has not been studied.

In most cases, conservative treatment is preferred for stable patients without ongoing pain $^{2}$. Those who present with myocardial ischemia with ongoing chest pain or hemodynamic instability should undergo revascularization especially when the dissection affects major arteries ${ }^{4}$. The immediate and long term success rate with PCI for SCAD is poor compared with atherosclerotic lesions. The immediate success rate of PCI is only about $65 \%^{5}$. The predominant reason for failure of PCI is 
inability to cross the guidewire. This usually happens in ostial or proximal lesions, as seen in our case .There are some strategies which can improve the outcome of PCI in such scenarios. We can use a hydrophilic wire with additional support of balloons or microcatheters to cross the lesion. For longer lesions, which requires multiple overlapping stents, we can first stent the distal edge, followed by the proximal edge and then the middle of the dissection to prevent propagation of the dissection ${ }^{3}$. Some authors have also advocated the use of longer stents for focal lesions for adequate coverage of both the edges of the dissection ${ }^{3}$, which we did in our case.

\section{REFERENCES}

[1] Vanzetto G, Berger-Coz E, Barone-Rochette G, et al. Prevalence, therapeutic management and medium-term prognosis of spontaneous coronary artery dissection: results from a database of 11,605 patients. Eur J Cardiothorac Surg 2009; 35: 250-4.

[2] Maeder M, Ammann P, Angehrn W, Rickli H. Idiopathic spontaneous coronary artery dissection: incidence, diagnosis and treatment. Int J Card 2005; 101: 363-69.

[3] Walsh SJ, Jokhi PP, Saw J. Successful percutaneous management of coronary dissection and extensive intramural haematoma associated with ST elevation MI. Acute Card Care 2008;10:231-3.

[4] Saw J, Aymong E, Sedlak T, et al. Spontaneous coronary artery dissection: association with predisposing arteriopathies and precipitating stressors and cardiovascular outcomes. Circ Cardiovasc Interv 2014;7:645-55.

[5] Tweet MS, Hayes SN, Pitta SR, et al. Clinical features, management, and prognosis of spontaneous coronary artery dissection. Circulation 2012;126:579-88 